Pacific Journal of Mathematics

REAL HOMOLOGY OF LIE GROUP HOMOMORPHISMS 


\title{
REAL HOMOLOGY OF LIE GROUP HOMOMORPHISMS
}

\author{
ROBERT F. BROWN
}

Let $h: G_{1} \rightarrow G_{2}$ be a homomorphism of compact, connected Lie groups and let $h_{*}: H_{*}\left(G_{1}\right) \rightarrow H_{*}\left(G_{2}\right)$ be the homomorphism of homology with real coefficients induced by $h$. The investigation of the properties of $h$ that can be deduced from a knowledge of $h_{*}$ goes back at least to work of Dynkin in the early 1950's. This paper presents several contributions to the investigation. The main result is a characterization of homomorphisms with abelian images as those whose induced homomorphisms annihilate all three-dimensional indecomposables. We then examine what the homology can tell us about the dimension of the abelian image. Next, an inequality relating the homology of the kernel of $h$ to the kernel of $h_{*}$ leads to sufficient conditions for $h$ to have an abelian, semisimple, or finite kernel. The final sections present various relationships between $h_{*}$ and the kernel and image of $h$ and, in particular, show that if $h\left(G_{1}\right)$ is totally nonhomologous to zero in $G_{2}$, then $h_{*}$ gives quite precise information about the behavior of $h$.

For the results of Dynkin, see [2] and [3].

In order to avoid frequently repeating the same hypotheses, we state that throughout this paper:

HYPOTHESES. $\quad h: G_{1} \rightarrow G_{2}$ is a homomorphism where $G_{1}$ and $G_{2}$ are compact, connected Lie groups.

1. Homomorphisms with abelian images. A homomorphism $h: G_{1} \rightarrow G_{2}$ induces homomorphisms of real homology $h_{* s}: H_{s}\left(G_{1}\right) \rightarrow$ $H_{s}\left(G_{2}\right)$ for all $s$. We will need the following observation:

Lemma 1.1. If $h: G_{1} \rightarrow G_{2}$ is surjective, then $h_{* s}: H_{s}\left(G_{1}\right) \rightarrow H_{s}\left(G_{2}\right)$ is surjective for all $s$.

Proof. Since $h$ is surjective, there is an isomorphism $\bar{h}: G_{1} / K \rightarrow$ $G_{2}$, where $K$ is the kernel of $h$. The quotient homomorphism $q: G_{1} \rightarrow G_{1} / K$ induces a surjection of real homology, so the commutativity of 


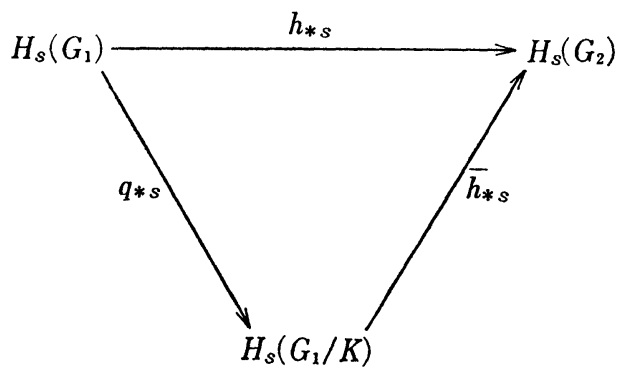

proves that $h_{* \varepsilon}$ is a surjection.

Let $Q H_{*}(G)$ denote the graded vector space of indecomposables in the real homology of a Lie group $G$. A homomorphism $h: G_{1} \rightarrow G_{2}$ induces $Q h_{* 8}: Q H_{8}\left(G_{1}\right) \rightarrow Q H_{8}\left(G_{2}\right)$ by restricting $h_{*}$ to the indecomposables in $H_{s}\left(G_{1}\right)$.

If a Lie group $G$ is abelian, then $Q H_{s}(G)=0$ for all $s>1$. Consequently, if $h: G_{1} \rightarrow G_{2}$ such that $h\left(G_{1}\right)$ is abelian, then certainly $Q h_{* s}=0$ for all $s>1$ because the homomorphism factors through $Q H_{*}\left(h\left(G_{1}\right)\right)$. But the converse holds as well; in fact our main result requires that we consider only $s=3$.

THEOREM 1.2. If $h: G_{1} \rightarrow G_{2}$ such that $Q h_{* 3}: Q H_{3}\left(G_{1}\right) \rightarrow Q H_{3}\left(G_{2}\right)$ is the zero homomorphism, then the group $h\left(G_{1}\right)$ is abelian.

Proof. We prove the contrapositive: assuming that $h\left(G_{1}\right)$ is not abelian we show that $Q h_{* 3} \neq 0$. Let $h^{\prime}: G_{1} \rightarrow h\left(G_{1}\right)$ be the same as $h$ except for range and let $j: h\left(G_{1}\right) \rightarrow G_{2}$ be inclusion. Consider the diagram

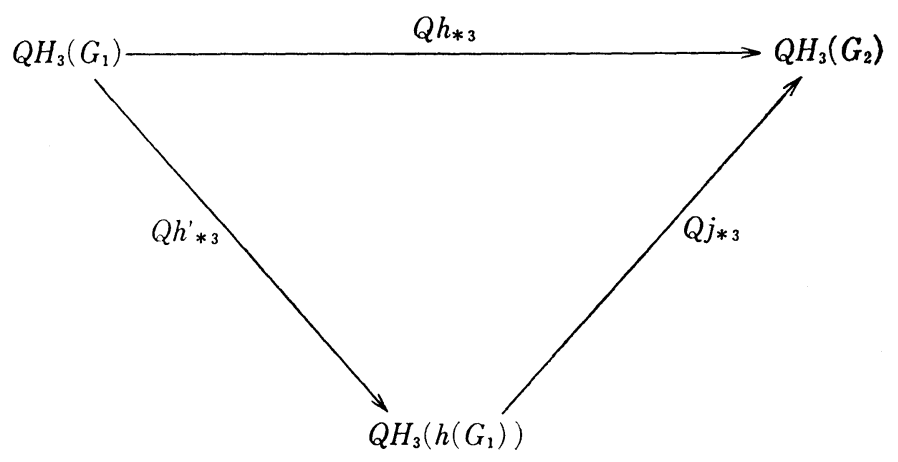

The homomorphism $Q h_{* 3}^{\prime}$ is surjective by Lemma 1.1 and the fact that the real homology of a Lie group is generated by indecomposables. Therefore, the diagram implies that $Q h_{* 3}=0$ if and only if $Q_{j * 3}=0$. So we will prove that if $h\left(G_{1}\right)$ is nonabelian then $Q_{j * 3} \neq 0$. Because $h\left(G_{1}\right)$ is assumed nonabelian, it is possible to exploit the relationship between compact and complex semisimple 
Lie groups to conclude that there is a closed subgroup $S$ of $h\left(G_{1}\right)$ that is semisimple and three-dimensional. Let $i: S \rightarrow G_{2}$ and $i^{\prime}: S \rightarrow$ $h\left(G_{1}\right)$ be inclusions, so we have the diagram

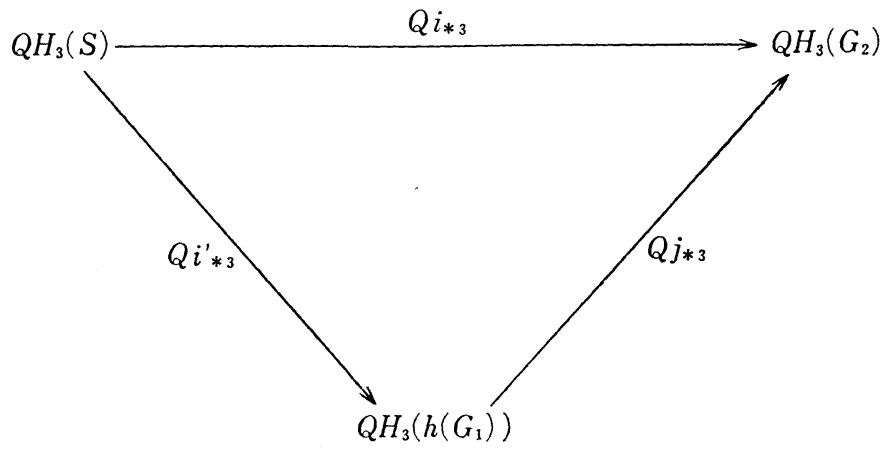

By [6], because $S$ is a semisimple and three-dimensional subgroup of $G_{2}$, then the homomorphism $i_{*}: H_{*}(S) \rightarrow H_{*}\left(G_{2}\right)$ is injective. Furthermore, the semisimplicity of $S$ implies $Q H_{3}(S) \neq 0$, so $Q j_{* 3} \neq$ 0 by the diagram and that completes the proof.

Two immediate consequences of the main result are:

CoROLlary 1.3. Suppose $h: G_{1} \rightarrow G_{2}$ where $G_{1}$ is semisimple. If $Q h_{* 3}=0$ then $h$ is the constant homomorphism.

CoRollary 1.4. If $Q h_{* 3}=0$ then $Q h_{* s}=0$ for all $s>1$.

Note that it is not sufficient in Corollary 1.3 merely to assume that $G_{1}$ is nonabelian. For instance, if we let $h: S^{1} \times S^{3} \rightarrow S^{3} \times S^{3}$ be the cartesian product of inclusion and the constant homomorphism, we obtain a nonconstant homomorphism for which $Q h_{* 3}=0$.

The homological characterization of homomorphisms with abelian images in Theorem 1.2 was of course suggested by the homological characterization: a compact Lie group $G$ is abelian if and only if $Q H_{3}(G)=0$. The characterization: a compact Lie group $G$ is semisimple if and only if $H_{1}(G)=0$ does not seem to lead to any characterization of homomorphisms with semisimple images. That is, the obvious condition $h_{* 1}=0$ is not sufficient for a semisimple image, as we can see by including abelian closed subgroups in simplyconnected Lie groups. (In contrast, the homological characterizations are precisely what we use to give sufficient conditions for $h$ to have an abelian or semisimple kernel in Corollaries 3.2 and 3.3 below.)

Problem. Find necessary and sufficient conditions on $h_{*}$ so 
that $h\left(G_{1}\right)$ is semisimple.

2. The dimension of an abelian image. Now that we have identified the homomorphisms with abelian images by means of their induced homology homomorphisms, we are led to investigate what homology can tell us about the only significant characteristic of such an image: its dimension.

It is convenient to recall the following concept from [5]. Let $A$ be a closed, connnected subgroup of a compact Lie group $G$, then $A$ is totally nonhomologous to zero in $G$, written $A \nsim 0$ in $G$, if and only if the homomorphism $j_{*}$ induced by the inclusion $j: A \rightarrow G$ is injective.

We have already made use of the concept in the proof of Theorem 1.2 because that proof depended on the fact that if $A$ is semisimple and three-dimensional, then $A \nsim 0$ in $G$.

We continue to use the notation $K$ for the kernel of $h: G_{1} \rightarrow G_{2}$ and denote by $K_{0}$ its maximal connected subgroup. We abbreviate dimension, of either a vector space or a Lie group, by dim, and image by im.

THEOREM 2.1. If the image of $h: G_{1} \rightarrow G_{2}$ is abelian, then

$$
\operatorname{dim} \operatorname{im} h_{* 1} \leqq \operatorname{dim} h\left(G_{1}\right) \leqq \operatorname{dim} H_{1}\left(G_{1}\right)
$$

with equality as follows:

(i) $\operatorname{dim} h\left(G_{1}\right)=\operatorname{dimim} h_{* 1}$ if and only if $h\left(G_{1}\right) \nsim 0$ in $G_{2}$

(ii) $\operatorname{dim} h\left(G_{1}\right)=\operatorname{dim} H_{1}\left(G_{1}\right)$ if and only if $K_{0}$ is semisimple.

Proof. Since $h\left(G_{1}\right)$ is a torus, $\operatorname{dim} h\left(G_{1}\right)=\operatorname{dim} H_{1}\left(h\left(G_{1}\right)\right)$. Defining $h^{\prime}: G_{1} \rightarrow h\left(G_{1}\right)$ and $j: h\left(G_{1}\right) \rightarrow G_{2}$ as before, we find that $\operatorname{dim} \operatorname{im} h_{* 1} \leqq$ $\operatorname{dim} \operatorname{im} h_{* 1}^{\prime}$ with equality if and only if $j_{* 1}$ is injective. Again using the fact that $h\left(G_{1}\right)$ is a torus, we conclude that $j_{* 1}$ is injective if and only if $h\left(G_{1}\right) \nsim 0$ in $G_{2}$. Since

$$
\operatorname{dim} H_{1}\left(G_{1}\right)=\operatorname{dim} H_{1}\left(K_{0}\right)+\operatorname{dim} H_{1}\left(h\left(G_{1}\right)\right)
$$

then $\operatorname{dim} h\left(G_{1}\right) \leqq \operatorname{dim} H_{1}\left(G_{1}\right)$ with equality if and only if $H_{1}\left(K_{0}\right)=0$.

CoROLlaRY 2.2. If the image of $h: G_{1} \rightarrow G_{2}$ is abelian and $h_{* 1}$ is injective, then $\operatorname{dim} h\left(G_{1}\right)=\operatorname{dim} Z\left(G_{1}\right)$, where $Z\left(G_{1}\right)$ is the center of $G_{1}$.

Proof. Since $h_{* 1}$ is injective, then $\operatorname{dim} \operatorname{im} h_{* 1}=\operatorname{dim} H_{1}\left(G_{1}\right)$ so $\operatorname{dim} h\left(G_{1}\right)=\operatorname{dim} H_{1}\left(G_{1}\right)$ by Theorem 2.1. The fact that $\operatorname{dim} H_{1}\left(G_{1}\right)=$ $\operatorname{dim} Z\left(G_{1}\right)$ completes the proof. 
3. The kernel of a homomorphism. Since we have seen that $Q h_{*}$ contains information about the image of $h: G_{1} \rightarrow G_{2}$, at least when the image is abelian, we may hope that $Q h_{*}$ will also reflect some of the properties of the kernel of $h$. We will show that to a certain extent this is indeed the case. Our results depend on the following useful inequality.

THEOREM 3.1. Let $h: G_{1} \rightarrow G_{2}$, then

$$
\operatorname{dim} Q H_{s}\left(K_{0}\right) \leqq \operatorname{dim} \operatorname{ker} Q h_{* s}
$$

for all $s>0$, where ker denotes kernel.

Proof. Let $i: K_{0} \rightarrow G_{1}$ be inclusion and let di: $\mathscr{K} \rightarrow \mathscr{S}_{1}$ be its differential, where $\mathscr{K}$ and $\mathscr{S}_{1}$ are the Lie algebras of $K_{0}$ and $G_{1}$ respectively. There is a projection $\pi: \mathbb{S}_{1} \rightarrow \Omega$ that is a Lie algebra homomorphism. Since $\pi(\mathrm{di})$ is the identity on $\Re$, the composition

$$
H_{s}(\mathscr{K}) \stackrel{(\mathrm{di})_{*_{s}}}{\longrightarrow} H_{s}\left(\mathbb{S}_{1}\right) \stackrel{\pi_{* s}}{\longrightarrow} H_{s}(\mathscr{K})
$$

is the identity for each $s$. We conclude that $(\mathrm{di})_{*_{s}}$ is an injection. Then the Universal Coefficient Theorem and de Rham's Theorem imply that $i_{* s}: H_{s}\left(K_{0}\right) \rightarrow H_{s}\left(G_{1}\right)$ is injective; as is the restriction $Q i_{* s}$. Since $h i$ is the constant homomorphism, the composition

$$
Q H_{s}\left(K_{0}\right) \stackrel{Q i_{* s}}{\longrightarrow} Q H_{s}\left(G_{1}\right) \stackrel{Q h_{* s}}{\longrightarrow} Q H_{s}\left(G_{2}\right)
$$

is the zero homomorphism for each $s>0$. Therefore $Q i_{*_{s}}\left(Q H_{s}\left(K_{0}\right)\right) \subseteq$ $\operatorname{ker} Q h_{* s}$ and because we have proved that $Q i_{* s}$ is injective

$$
\operatorname{dim} Q H_{s}\left(K_{0}\right)=\operatorname{dim} \operatorname{im} Q i_{* s} \leqq \operatorname{dim} \operatorname{ker} Q h_{* s} .
$$

For an example where $\operatorname{dim} Q H_{s}\left(K_{0}\right) \neq \operatorname{dim} \operatorname{ker} Q h_{* s}$, let $h: S O(8) \rightarrow$ $S O(9)$ be inclusion. The Wang sequence of the fibration

$$
S O(8) \stackrel{h}{\longrightarrow} S O(9) \longrightarrow S^{8}
$$

shows that $\operatorname{dim} \operatorname{ker} Q h_{* r} \geqq 1$.

CoROLlaRY 3.2. If $h_{* 1}$ is injective, then $K_{0}$ is semisimple.

Proof. Since $Q h_{* 1}=h_{* 1}$, then $\operatorname{dim} \operatorname{ker} Q h_{* 1}=0$ so $H_{1}\left(K_{0}\right)=0$ by Theorem 3.1 and therefore $K_{0}$ is semisimple.

The injectivity of $h_{* 1}$ is sufficient for $K_{0}$ to be semisimple, but it is obvious that it is not a necessary condition.

CoROLlaRy 3.3. If $Q h_{* 3}$ is injective, then $K_{0}$ is abelian. 
Once again, injectivity is a sufficient but not necessary condition. Define $h: \operatorname{Sp}(6) \times \operatorname{Sp}(6) \rightarrow \operatorname{Sp}(12)$ as follows. Given $A$ and $B$ in $\operatorname{Sp}(6)$, let $h(A, B)$ be the matrix

$$
\left[\begin{array}{cc}
A & 0 \\
0 & B
\end{array}\right]
$$

Then $h$ has trivial kernel, but $Q h_{* 3}$ cannot be injective because $\operatorname{dim} Q H_{3}(\operatorname{Sp}(6) \times \operatorname{Sp}(6))=2$ and $\operatorname{dim} Q H_{3}(\operatorname{Sp}(12))=1$.

Combining the previous corollaries, we see that if $h_{* 1}$ and $h_{* 3}$ are both injective, then $K_{0}$ is trivial so

CoROLLARY 3.4. If $h_{* s}$ is injective for $s \leqq 3$, then the kernel of $h$ is finite.

In common with the previous corollaries, the converse to Corollary 3.4 is not true in general. However

THEOREM 3.5. Suppose $h: G_{1} \rightarrow G_{2}$ has finite kernel. Then $h_{*}$ is injective if and only if $h\left(G_{1}\right) \nsim 0$ in $G_{2}$.

Proof. Let $h^{\prime}: G_{1} \rightarrow h\left(G_{1}\right)$ be the same as $h$ except for range. Since $h^{\prime}$ has finite kernel, its differential $d h^{\prime}: \mathbb{S}_{1} \rightarrow \mathfrak{K}$ is an isomorphism $\left(\mathbb{S}_{1}\right.$ and $\mathscr{S}$ are the Lie algebras of $G_{1}$ and $h\left(G_{1}\right)$, respectively). Therefore, $\left(d h^{\prime}\right)_{*}: H_{*}\left(\mathbb{S}_{1}\right) \rightarrow H_{*}\left(\mathfrak{F}_{\mathcal{E}}\right)$ is an isomorphism and it follows that $h_{*}^{\prime}: H_{*}\left(G_{1}\right) \rightarrow H_{*}\left(h\left(G_{1}\right)\right)$ is an isomorphism also. For $j: h\left(G_{1}\right) \rightarrow G_{2}$ the inclusion, the commutativity of

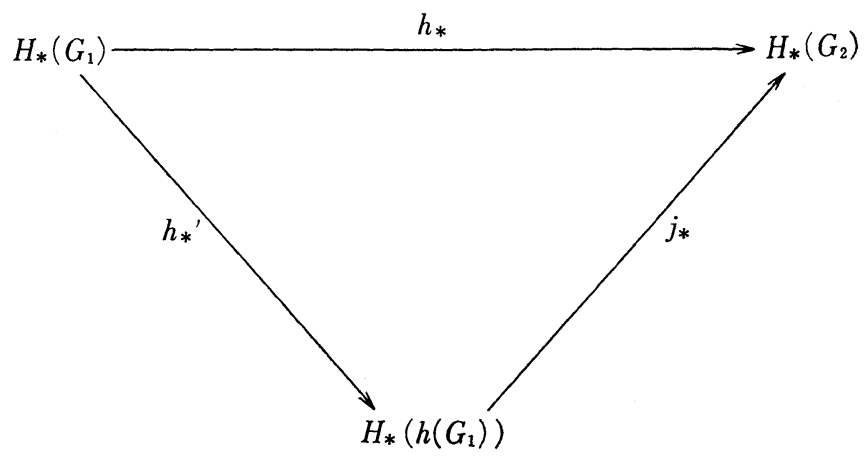

implies that $h_{*}$ is injective if and only if $j_{*}$ is, that is, if and only if $h\left(G_{1}\right) \nsim 0$ in $G_{2}$.

4. Rank and dimension of the kernel. We have seen in the previous section that $Q h_{*}$ does contain information about the kernel $K$ of $h: G_{1} \rightarrow G_{2}$, at least to the extent of producing sufficient condi- 
tions for $K$ to be semisimple or abelian. In this section, we improve a result from [1] to obtain upper bounds on the rank and dimension of $K$. Furthermore, we find a necessary and sufficient condition for the bounds to provide exact calculations.

The rank of a compact Lie group will be denoted by $\operatorname{rk}(G)$. (If $G$ is disconnected, its rank is defined to be the rank of the maximal connected subgroup of $G$.) The dimension of a graded vector space, still denoted by dim, is the sum of the dimensions of the individual vector spaces.

Since the bounds on the rank and dimension of $K$ are most naturally stated in the language of cohomology rather than homology, we consider the homomorphisms $h^{* s}: H^{s}\left(G_{2}\right) \rightarrow H^{s}\left(G_{1}\right)$, for each $s$, induced by $h: G_{1} \rightarrow G_{2}$ on cohomology with real coefficients. Restricting to primitives, we have homomorphisms $P h^{* s}: P H^{s}\left(G_{2}\right) \rightarrow$ $P H^{s}\left(G_{1}\right)$.

THEOREM 4.1. Let $K$ be the kernel of $h: G_{1} \rightarrow G_{2}$, then

(i) $\operatorname{rk}(K) \leqq \operatorname{dim} \operatorname{ker} P h^{*}+\left(\operatorname{rk}\left(G_{1}\right)-\operatorname{rk}\left(G_{2}\right)\right)$

(ii) $\operatorname{dim}(K) \leqq \sum_{s} s\left(\operatorname{dim} \operatorname{ker} P h^{* 8}\right)+\left(\operatorname{dim}\left(G_{1}\right)-\operatorname{dim}\left(G_{2}\right)\right)$.

The inequalities (i) and (ii) are equalities if and only if $h\left(G_{1}\right) \nsim 0$ in $G_{2}$.

Proof. Since $K$ is a normal subgroup of $G_{1}$, the quotient homomorphism $q: G_{1} \rightarrow G_{1} / K$ induces an injective homomorphism of real cohomology, so $P q^{* s}$ is injective for all $s$. From the commutative diagram below, where $\bar{h}$ is an isomorphism and $h^{\prime}$ is the same as $h$ except for range, we see that $P h^{\prime * s}$ is injective because $P q^{* s}$ is.

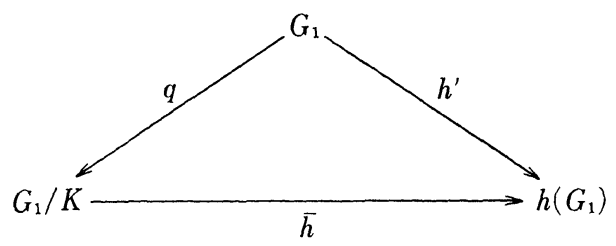

Therefore, the diagram

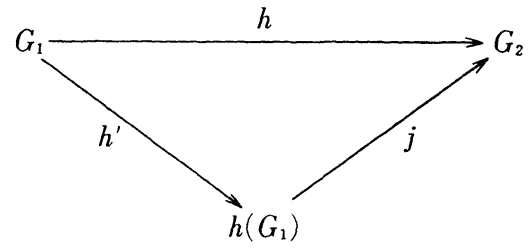

where $j$ is inclusion, implies that $\operatorname{ker} P h^{* s}=\operatorname{ker} P j^{* s}$ and thus since $\operatorname{dim} P H^{s}\left(G_{2}\right)=\operatorname{dim} \operatorname{ker} P j^{* s}+\operatorname{dim} i m P j^{* s}$ 
that

(*) $\quad \operatorname{dim} \operatorname{ker} P h^{* s}-\operatorname{dim} P H^{s}\left(G_{2}\right)+\operatorname{dim} i m P j^{* s}=0$.

Now, for the proof of part (i), we know from [4] that for any compact Lie group, $\operatorname{rk}(G)=\operatorname{dim} P H^{*}(G)$. Since

$$
P H^{*}\left(G_{1}\right)=P H^{*}(K) \oplus P H^{*}\left(h\left(G_{1}\right)\right)
$$

we see that

$$
\operatorname{rk}\left(G_{1}\right)=\operatorname{rk}(K)+\operatorname{rk}\left(h\left(G_{1}\right)\right) .
$$

Using $\left(^{*}\right)$ and rearranging terms, we have

$$
\begin{aligned}
\operatorname{rk}(K)= & {\left[\operatorname{dim} \operatorname{ker} P h^{*}+\left(\operatorname{rk}\left(G_{1}\right)-\operatorname{rk}\left(G_{2}\right)\right)\right] } \\
& -\left[\operatorname{rk}\left(h\left(G_{1}\right)\right)-\operatorname{dim} \operatorname{im} P j^{*}\right] .
\end{aligned}
$$

Inequality (i) follows because im $P j^{*} \cong P H^{*}\left(h\left(G_{1}\right)\right.$ ). We have equality in (i) if and only if $P j^{*}$ is surjective which, by the Universal Coefficient Theorem, is equivalent to the condition $h\left(G_{1}\right) \nsim 0$ in $G_{2}$. For part (ii) we use the fact that for any compact, connected lie group

$$
\operatorname{dim}(G)=\sum_{s} s\left(\operatorname{dim} P H^{s}(G)\right) \text {. }
$$

Since

$$
\operatorname{dim}\left(G_{1}\right)=\operatorname{dim}(K)+\operatorname{dim}\left(h\left(G_{1}\right)\right)
$$

we again use $\left(^{*}\right)$ and rearrange terms to conclude that

$$
\begin{aligned}
\operatorname{dim}(K)= & {\left[\sum_{s} s\left(\operatorname{dim} \operatorname{ker} P h^{* s}\right)+\left(\operatorname{dim}\left(G_{1}\right)-\operatorname{dim}\left(G_{2}\right)\right)\right] } \\
& -\left[\operatorname{dim}\left(h\left(G_{1}\right)\right)-\sum_{s} s\left(\operatorname{dim} \operatorname{im} P j^{* s}\right)\right] .
\end{aligned}
$$

Just as in the proof of part (i), inequality (ii) follows from this last equation, with equality in (ii) if and only if $h\left(G_{1}\right) \nsim 0$ in $G_{2}$.

The less precise form of Theorem 4.1(i) in [1] was used there to study homomorphisms $h$ for which $h^{*}$ is an isomorphism.

CoRollary 4.2. If the image of $h: G_{1} \rightarrow G_{2}$ is abelian, then

(i) $\operatorname{rk}(K) \leqq \operatorname{rk}\left(G_{1}\right)-\operatorname{dim} \operatorname{im} h^{* 1}$

(ii) $\operatorname{dim}(K) \leqq \operatorname{dim}\left(G_{1}\right)-\operatorname{dim} \operatorname{im} h^{* 1}$ with equality if and only if $h\left(G_{1}\right) \nsim 0$ in $G_{2}$.

Proof. We will prove inequality (i); the proof of (ii) is almost identical. Since $h\left(G_{1}\right)$ abelian implies $h_{* s}=0$ for $s>1$ the Universal Coefficient Theorem implies that $h^{* s}=0$ for $s>1$ also and thus

$$
\operatorname{dim} \operatorname{ker} P h^{*}=\operatorname{dim} \operatorname{ker} P h^{* 1}+\sum_{s>1} \operatorname{dim} P H^{s}\left(G_{2}\right) .
$$


Because

$$
\operatorname{rk}\left(G_{2}\right)=\operatorname{dim} H^{1}\left(G_{2}\right)+\sum_{s>1} \operatorname{dim} P H^{s}\left(G_{2}\right)
$$

we may substitute into inequality (i) of Theorem 4.1 to conclude that

$$
\operatorname{rk}(K) \leqq \operatorname{rk}\left(G_{1}\right)-\left(\operatorname{dim} H^{1}\left(G_{2}\right)-\operatorname{dim} \operatorname{ker} h^{* 1}\right) .
$$

We can apply Corollary 4.2 quite effectively when $G_{2}$ is abelian. For instance

CoROLlaRy 4.3. Suppose $h: G_{1} \rightarrow G_{2}$ is a homomorphism and $G_{2}$ is abelian, then

$$
\operatorname{dim}(K)=\left(\operatorname{dim}\left(G_{1}\right)-\operatorname{dim}\left(G_{2}\right)\right)+\operatorname{dim} \operatorname{ker} h^{* 1} .
$$

5. Surjective homomorphisms. The following homological characterization of surjective homomorphisms is easy to verify, but we include it for completeness.

Proposition 5.1. A homomorphism $h: G_{1} \rightarrow G_{2}$ is surjective if and only if $h_{* n}: H_{n}\left(G_{1}\right) \rightarrow H_{n}\left(G_{2}\right)$ is a nonzero homomorphism, where $n=\operatorname{dim}\left(G_{2}\right)$.

Proof. Necessity is immediate from Lemma 1.1 and the fact that since $G_{2}$ is a closed orientable $n$-manifold, $H_{n}\left(G_{2}\right) \neq 0$. Sufficiency is a consequence of the classical result that if a map of closed orientable manifolds has nonzero degree, then it is surjective. Alternatively, we may prove sufficiency using Theorem 4.1(ii) as follows. If $h_{* n} \neq 0$ then $h^{* n}: H^{n}\left(G_{2}\right) \rightarrow H^{n}\left(G_{1}\right)$ is nonzero. Let $z_{1}, z_{2}, \cdots, z_{r}$ generate the exterior algebra $H^{*}\left(G_{2}\right)$, then $\mu=z_{1} z_{2} \cdots z_{r}$ generates the vector space $H^{n}\left(G_{2}\right)$. We are assuming $h^{*}(\mu) \neq 0$ which implies $P h^{*}$ is injective. By inequality (ii) of Theorem 4.1, $\operatorname{dim}(K) \leqq \operatorname{dim}\left(G_{1}\right)-\operatorname{dim}\left(G_{2}\right)$. But then we have

$$
\operatorname{dim}\left(G_{1}\right)-\operatorname{dim}\left(h\left(G_{1}\right)\right) \leqq \operatorname{dim}\left(G_{1}\right)-\operatorname{dim}\left(G_{2}\right) .
$$

Since $h\left(G_{1}\right)$ is a closed submanifold of $G_{2}$, it must be that $h\left(G_{1}\right)=G_{2}$, that is, $h$ is surjective.

\section{REFERENCES}

1. R. Brown, Cohomology of homomorphisms of Lie algebras and Lie groups, Pacific J. Math., 69 (1977), 325-332.

2. E. Dynkin, $A$ connection between homologies of a compact Lie group and its subgroups, Doklady Akad. Nauk SSSR (N.S.), 87 (1952), 333-336. 
3. E. Dynkin, Topological characteristics of homomorphisms of compact Lie groups, Amer. Math. Soc. Tranl. (2), 12 (1959), 301-342.

4. H. Hopf, Über den Rang geschlossener Liescher Gruppen, Comment. Math. Helv., 13 (1940), 119-143.

5. H. Samelson, Beiträge zur Topologie der Gruppen-Mannigfaltigkeiten, Ann. of Math. (2), 42 (1941), 1091-1137.

6. - Sur les sous-groupes de dimension 3 des groupes de Lie compacts, C.R. Acad. Sci. Paris, 228 (1949), 630-631.

Received September 3, 1980.

UNIVERSITY OF WARWICK

Coventry, England

AND

UNIVERsity of CALIFornia, Los ANGEleS

Los ANGELS, CA 90024 


\section{PACIFIC JOURNAL OF MATHEMATICS}

\section{EDITORS}

DONALD BABBITT (Managing Editor)

University of California

Los Angeles, California 90024

Hugo RossI

University of Utah

Salt Lake City, UT 84112

C. C. MOORE and ARTHUR Agus

University of California

Berkeley, CA 94720

\section{J. DUGUNDJI}

Department of Mathematics University of Southern California Los Angeles, California 90007

R. FinN and J. MILGRAM Stanford University Stanford, California 94305

\section{ASSOCIATE EDITORS}
R. ARNES
E. F. BeCKENBACH
B. H. Neumann
F. WOLF
K. YosHIDA

\section{SUPPORTING INSTITUTIONS}

UNIVERSITY OF ARIZONA

UNIVERSITY OF BRITISH COLUMBIA

CALIFORNIA INSTITUTE OF TECHNOLOGY

UNIVERSITY OF CALIFORNIA

MONTANA STATE UNIVERSITY

UNIVERSITY OF NEVADA, RENO

NEW MEXICO STATE UNIVERSITY

OREGON STATE UNIVERSITY
UNIVERSITY OF OREGON UNIVERSITY OF SOUTHERN CALIFORNIA STANFORD UNIVERSITY UNIVERSITY OF HAWAII UNIVERSITY OF TOKYO UNIVERSITY OF UTAH WASHINGTON STATE UNIVERSITY UNIVERSITY OF WASHINGTON 


\section{Pacific Journal of Mathematics}

Vol. 100, No. $1 \quad$ September, 1982

Charalambos D. Aliprantis, Owen Sidney Burkinshaw and M. Duhoux,

Compactness properties of abstract kernel operators $\ldots \ldots \ldots \ldots \ldots \ldots 1$

Roger C. Alperin, Locally compact groups acting on trees .............23

Robert F. Brown, Real homology of Lie group homomorphisms ......... 33

Karen Chase, Maximal groups in sandwich semigroups of binary relations . . 43

W. Wistar (William) Comfort and T. Soundararajan, Pseudocompact

group topologies and totally dense subgroups $\ldots \ldots \ldots \ldots \ldots \ldots \ldots 61$

M. Ferri and C. Gagliardi, Crystallisation moves $\ldots \ldots \ldots \ldots \ldots \ldots \ldots$

Kenneth R. Goodearl, Directly finite aleph-nought-continuous regular

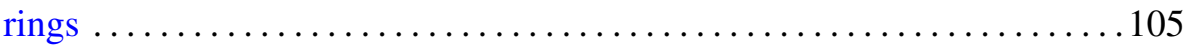

Edward Lewis Green, On the representation theory of rings in matrix

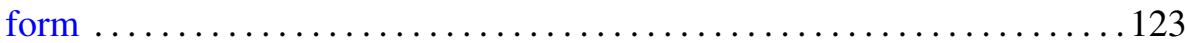

Walter Hengartner and Glenn E. Schober, Interpolation, continuation, and

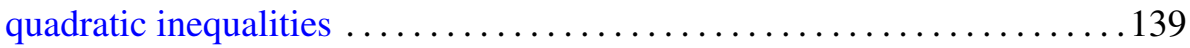

Kenneth Kunen and Haskell Paul Rosenthal, Martingale proofs of some geometrical results in Banach space theory $\ldots \ldots \ldots \ldots \ldots \ldots \ldots \ldots \ldots \ldots$

Brian William McEnnis, Shifts on indefinite inner product spaces. II . . . . . 177

Roman Pol, Note on the spaces $P(S)$ of regular probability measures whose topology is determined by countable subsets $\ldots \ldots \ldots \ldots \ldots \ldots \ldots \ldots 185$

Joan Manuel Verdera Melenchón, Finitely generated projective extensions

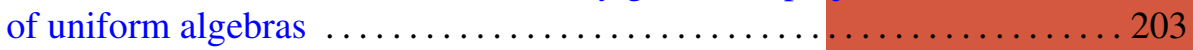

Cheng Ye You, Fixed point classes of a fiber map .................. 217 